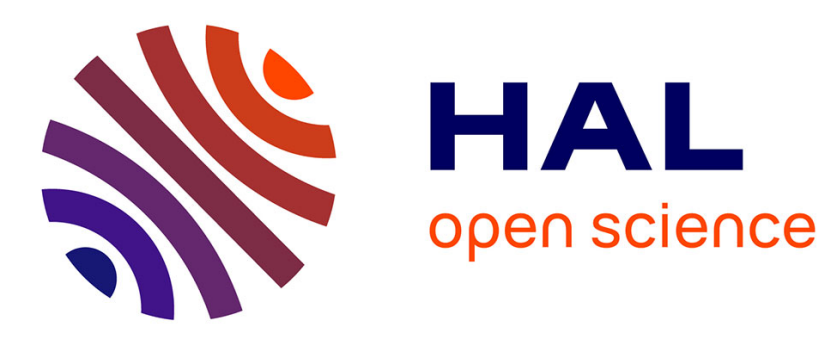

\title{
Confinement of Vibrotactile Stimuli in Narrow Plates
} Ayoub Ben Dhiab, Charles Hudin

\section{To cite this version:}

Ayoub Ben Dhiab, Charles Hudin. Confinement of Vibrotactile Stimuli in Narrow Plates. 2019 IEEE World Haptics Conference (WHC), Jul 2019, Tokyo, Japan. pp.431-436, 10.1109/WHC.2019.8816081 . cea-02298419

\section{HAL Id: cea-02298419 https://hal-cea.archives-ouvertes.fr/cea-02298419}

Submitted on 26 Sep 2019

HAL is a multi-disciplinary open access archive for the deposit and dissemination of scientific research documents, whether they are published or not. The documents may come from teaching and research institutions in France or abroad, or from public or private research centers.
L'archive ouverte pluridisciplinaire HAL, est destinée au dépôt et à la diffusion de documents scientifiques de niveau recherche, publiés ou non, émanant des établissements d'enseignement et de recherche français ou étrangers, des laboratoires publics ou privés. 


\title{
Confinement of Vibrotactile Stimuli in Narrow Plates.
}

\author{
Ayoub Ben Dhiab ${ }^{1}$, Charles Hudin ${ }^{1}$
}

\begin{abstract}
On a touch surface, providing a local vibrotactile feedback enables multiusers and multitouch interactions. While the vibration propagation usually impedes this localization, we show in this paper that narrow strip-shaped plates constitute waveguides in which bending waves below a cut-off frequency do not propagate. We provide a theoretical explanation of the phenomenon and experimental validations. We thus show that vibrations up to $2 \mathrm{kHz}$ are well confined on top of the actuated area with vibration amplitude over $1 \mu \mathrm{m}$ that can be felt by the fingers. The principle was validated with piezoelectric actuators of various shapes and a vibration motor.
\end{abstract}

\section{INTRODUCTION}

Vibrations within the tactile sensitivity range of frequency are used to convey information, but also, when correlated with finger motion and force, to simulate clicks [1], [2], bumps, texture or compliance [3], [4]. Wave propagation and reverberation effects usually lead to a uniform repartition of vibration energy across the surface. Actuators, acting as local sources of vibration, produce a global and uniform stimulation. Providing different feedback at different positions is then only achievable for single finger exploration through a temporal variation correlated to the finger position [5]. This approach however raises two issues. Firstly, the whole contact area experiences the same vibration thus making it impossible to display, for a static finger, variations at a scale smaller than finger size. Secondly, is the impossibility to provide distinct feedback to multiple fingers. The vibrotactile feedback provided on standard interactive surfaces is therefore irrelevant for multitouch or multiuser interactions. Those two issues can be solved by localizing the vibration, that is by ensuring that vibration produced at a chosen position cannot be felt at an other. Existing approaches for localized haptics can be sorted in two types: on one hand, wavefield shaping approaches like Time Reversal [6], [7], [8], Inverse Filter [9], Modal Synthesis [10], [11] or Airborn Ultrasound [12] use signal processing and control strategies to create high amplitude vibrations at specific positions while maintaining vibration level low at others. On the other hand, confinement approaches like non-radiating frequencies [13] and patterned or damped surfaces [14] prevent vibrations from propagating outside of the actuated area. The work presented here is of the second type as it relies on the geometry of the propagation surface to confine vibrations within the actuated area, and does not involve any specific signal processing. This approach, however, differs from the non radiating approach in three ways. First, it allows for the localization of

\footnotetext{
${ }^{1}$ Ayoub Ben Dhiab and Charles Hudin are with the CEA, LIST, F-91191 Gif-sur-Yvette, France. ayoub.ben-dhiabdcea.fr; charles.hudin@cea.fr
}

low frequencies (below a few kilohertz) while non-radiating frequencies are ultrasonic frequencies well-suited for friction modulation only [15]. Second, the non-propagating behavior is observed over the whole bandwidth from DC to the cutoff frequency and not at specific discrete frequencies. Finally, the vibration confinement results from the surface geometry and boundary conditions and not from the actuator geometry. As a consequence, the method presented in this paper works equally well for piezoelectric actuators of different shapes and for electromagnetic vibration motors, as will be shown in the experimental validation. This method is however limited to narrow plates with width about $3 \mathrm{~cm}$. It is therefore not well-suited for screen but is very effective for 1D tactile interfaces like slidebars or single row keyboards.

\section{PRINCIPLE}

In this section, we explain how non-propagative vibrations can be obtained at low frequencies using the geometric properties of the waveguide. A waveguide is a medium where the wave propagation is bounded in two directions of space and free in the third one [16, Chapter 24]. They find applications in different fields of wave physics [17]. Electromagnetic waveguides are used as filters in radiocommunication. Single and multimode fiber optics rely on propagation modes to convey information [18]. Acoustic waveguides are used to design horns and high frequency electronic filters [19]. In our case the propagation medium is a thin plate with finite thickness and width but with an arbitrary length. The propagation of waves in a waveguide can be described in terms of propagation modes. Each of these modes is defined by its cross section profile, wave velocity and cut-off frequency [20, p. 201]. While in most waveguides the zero order propagation mode, with a cut-off frequency equal to $0 \mathrm{~Hz}$, exist. We show that in the case of a simply supported or clamped plate that zero-th has a null amplitude and therefore does not contribute to the propagation. As a result, bending waves cannot propagate in such guide below the cut-off frequency of the first propagation mode. We derive the expression of this frequency and show that in practical cases it can be greater than the frequency bandwidth of tactile stimuli.

\section{A. Theory}

The equation governing the transverse motion $u(x, y, t)$ of an isotropic homogeneous plate of constant thickness $h$ is given as [21, p. 233]:

$$
\rho h \frac{\partial^{2} u}{\partial t^{2}}+D \nabla^{4} u=0
$$




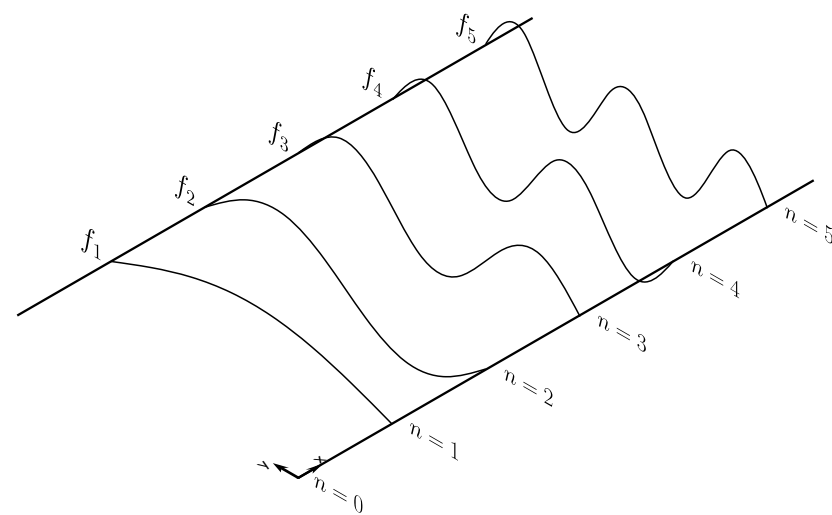

Fig. 1. First five propagation mode shape. Before $n=1$, only evanescent waves are produced. Then, we have a transition between propagative and evanescent behaviors.

with $\rho$ the mass density, $D$ the bending stiffness and $\nabla^{4}$ the biharmonic operator. Under Kirchhoff thin plate assumptions, $D$ can be expressed as $[21$, p. 233]:

$$
D=\frac{E h^{3}}{12\left(1-\nu^{2}\right)}
$$

where $E$ and $\nu$ are the Young's modulus and Poisson's ratio. Because we consider an homogeneous system, with constant mechanical and dimensional properties and constant boundary conditions, the solutions of (1) are invariant with respect to space and time translation. As a consequence we can separate variables and write:

$$
u(x, y, t)=\Psi(x) \Phi(y) \Theta(t)
$$

For harmonic behavior and waves going toward increasing $\mathrm{x}$, the response becomes $u(x, y, t)=\Phi(y) e^{j p x} e^{-j \omega t}$, where $\omega$ is the circular frequency and $p$ the wavenumber along the $\mathrm{x}$-axis. Putting this expression in (1) yields to the following differential equation for $\Phi(y)$ :

$$
\frac{\partial^{4} \Phi}{\partial y^{4}}-p^{2} \frac{\partial^{2} \Phi}{\partial y^{2}}+\left(p^{4}-k^{4}\right) \Phi=0
$$

where $k^{4}=\frac{\rho h}{D} \omega^{2}$ is the wave number and $p$ and $q$ are its components along $\mathrm{x}$-axis and $\mathrm{y}$-axis respectively. Therefore we have: $k^{2}=p^{2}+q^{2}$. A solution to that equation would be:

$$
\Phi(y)=C_{1} e^{-j q y}+C_{2} e^{j q y}+C_{3} e^{-q y}+C_{4} e^{q y}
$$

Considering the bounding conditions for a simply supported plate:

$$
\mid \begin{gathered}
u(x, y, t)=0 \\
\frac{\partial^{2} u(x, y, t)}{\partial y^{2}}=0
\end{gathered} \text { for } y=0, W
$$

where $W$ is the plate width. We find that:

$$
\Phi(y)=2 i \sin \left(q_{n} y\right)
$$

with:

$$
q_{n}=\frac{n \pi}{W}
$$

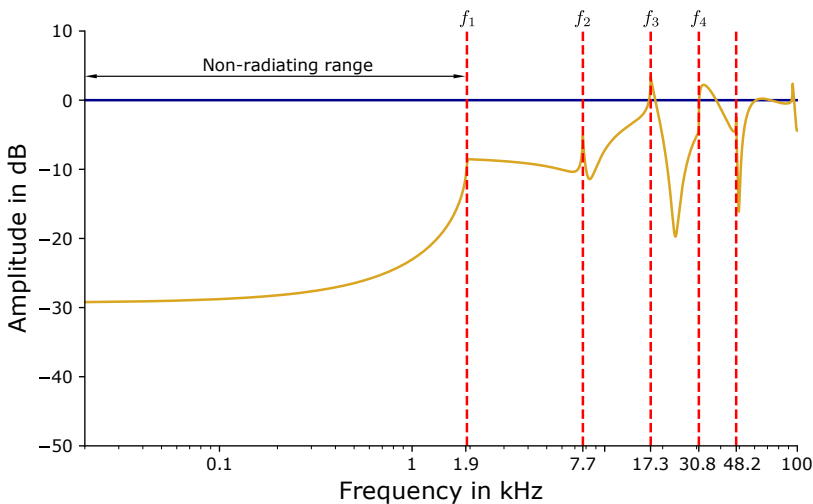

Fig. 2. Numerical solutions of equation (13) in the frequency domain at the center of the actuator (dark blue) and at a point $20 \mathrm{~mm}$ away on the $\mathrm{x}$-axis and at a $y=\frac{W}{6}$ from the actuator (light yellow). Frequencies from 0 to $1.9 \mathrm{kHz}$ are said to be non-propagative. At these frequencies, amplitudes at the center of the actuator are theoretically way higher than elsewhere on the plate. After the first cut-off frequency modes begin to propagate.

where $n$ is an integer corresponding to the mode index. The profile of modes with index 0 to 5 is depicted in Fig. 1. This solution yields to:

$$
p_{n}=\left(\frac{\rho h}{D}\right)^{\frac{1}{4}} \sqrt{\omega-\omega_{n}}
$$

with:

$$
\omega_{n}=\sqrt{\frac{D}{\rho h}} q_{n}^{2}=\sqrt{\frac{D}{\rho h}}\left(\frac{n \pi}{W}\right)^{2} .
$$

In terms of frequency we thus have:

$$
f_{n}=\frac{\omega_{n}}{2 \pi}=\frac{\pi}{4 \sqrt{3}} \frac{h}{W^{2}} \sqrt{\frac{E}{\rho\left(1-\nu^{2}\right)}} n^{2} .
$$

Now, when the circular frequency verifies $\omega<\omega_{n}$, the wavenumber $p_{n}$ becomes an imaginary number and turns the term $e^{j p_{n} x}$ into a decreasing exponential, with a decay constant:

$$
\alpha_{n}=-j p_{n}=\left(\frac{\rho h}{D}\right)^{\frac{1}{4}} \sqrt{\omega_{n}-\omega} \text { with } \omega<\omega_{n}
$$

The circular frequency $\omega_{n}$ is therefore a cut-off frequency that marks the transition between propagative and evanescent behaviors. The overall displacement is obtained by summing the individual contributions of propagation modes:

$$
u(x, y, t)=\left(\sum_{n=1}^{\infty} A_{n} \sin \left(q_{n} y\right) e^{j p_{n} x}\right) e^{-j \omega t}
$$

where $A_{n}$ is the complex amplitude of each mode. In our case, we aim to produce a local vibration field around the excitation point. Such a situation occurs when all modes are evanescent, that is for all frequencies lower than the first cut-off frequency $f_{1}$ :

$$
f_{1}=\frac{\pi}{4 \sqrt{3}} \frac{h}{W^{2}} \sqrt{\frac{E}{\rho\left(1-\nu^{2}\right)}}
$$




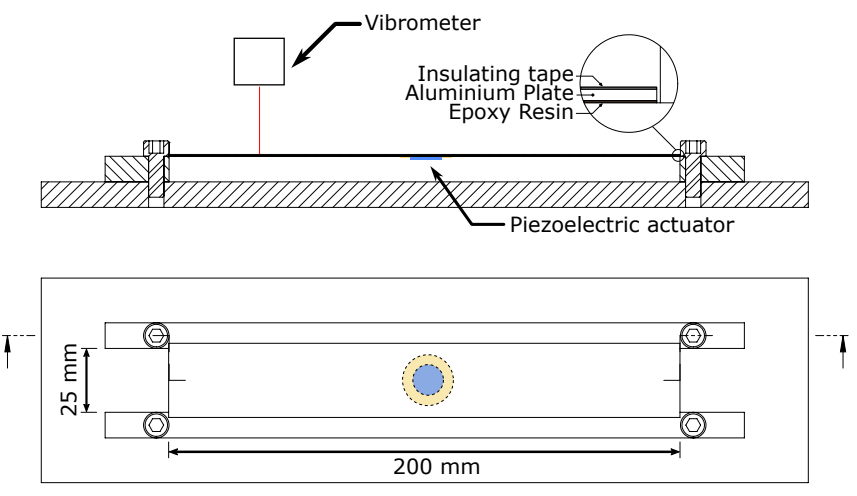

Fig. 3. Experimental setup. Top: A piezoelectric actuator is glued to the bottom side of an aluminum plate. The laser vibrometer measures the surfaces out of plane displacement. Bottom: plate dimensions and actuator position.

\section{B. Implementation}

Taking equation (13) with a plate of width $W=25 \mathrm{~mm}$, thickness $h=0.5 \mathrm{~mm}$ and aluminum properties $(\nu=0.346$, $E=67 \mathrm{MPa}, \rho=2700 \mathrm{~kg} / \mathrm{m}^{2}$ ) and mode amplitude $A_{n}=1$ gives us the frequency response function in Fig. 2.

The activation of the first mode at $1.9 \mathrm{kHz}$ leaves a nonpropagative range from 0 to $1.9 \mathrm{kHz}$. In other words, the geometry of the waveguide allows us to create only evanescent waves until the $1.9 \mathrm{kHz}$ cut-off frequency. Those evanescent waves, which amplitudes decrease exponentially with the source distance, can be used to give a localized vibrotactile feedback. After that cut-off frequency, modes begin to spread and the localized effect is lost.

Thanks to that non-propagative range, localized vibrotactile feedbacks can be confined to the actuated region of the surface.

\section{EXPERIMENTAL VALIDATION}

\section{A. Single piezoelectric actuator}

In order to validate the theory introduced in Section II, an aluminum plate with a piezoelectric actuator glued to the center of the bottom side was prepared. The actuator (muRata 7BB-20-3), of circular geometry, is composed of two part: a plate of diameter $20 \mathrm{~mm}$ and a thickness of $0.1 \mathrm{~mm}$ and an electrode of diameter $12.8 \mathrm{~mm}$ and a thickness of $0.11 \mathrm{~mm}$. The simply supported conditions being quite difficult to achieve, the plate, measuring $25 \times 200 \times 0.5$ $\mathrm{mm}^{3}$, was rather clamped on its longer sides to a rigid frame via epoxy resin and left free on both ends. This leads to several differences compared to the simply supported case. With clamped conditions: the shape of the deformation along the $y$-axis of the plate changes; the amplitude of vibration is less important; and the cut-off frequency is higher. The out of plane displacement of the surface is measured by a laser vibrometer (Polytec OFV-534/2570) mounted on a motorized 3 axis platform. This setup is illustrated on Fig. 3. The actuator was driven by an analog signal with $50 \mathrm{~V}$ peakto-peak amplitude. We measured the frequency response

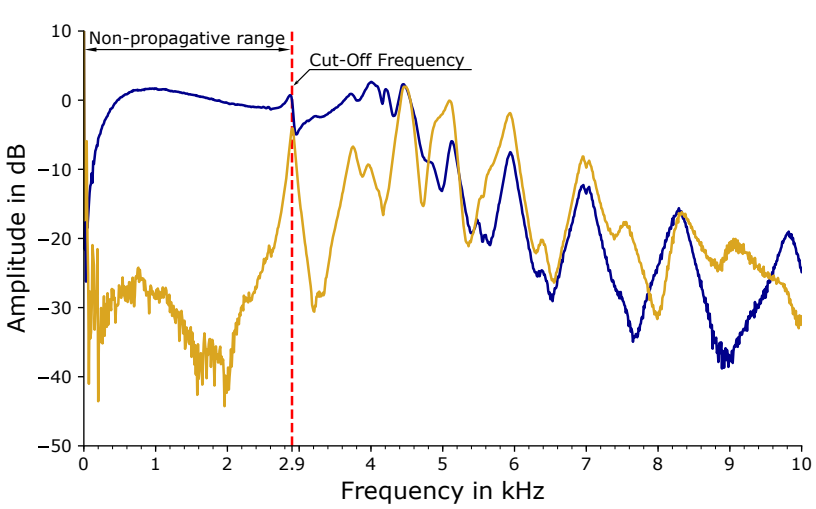

Fig. 4. Measured frequency response function at the center of the actuator (dark blue) and at a point $20 \mathrm{~mm}$ away from the actuator on the $\mathrm{x}$-axis (light gold). The frequency range between 0 and $2.9 \mathrm{kHz}$ is non-propagative. Within this range, amplitudes at the center of the actuator are about $30 \mathrm{~dB}$ larger than elsewhere on the plate.

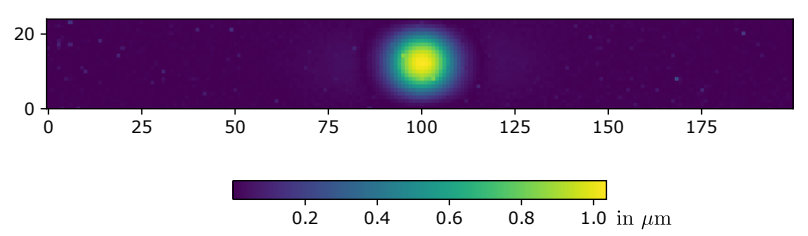

Fig. 5. Out of plane displacement in $\mu \mathrm{m}$ of the plate $\left(200 \times 25 \times 0.5 \mathrm{~mm}^{3}\right)$ at $300 \mathrm{~Hz}$. The area above the piezoelectric actuator has an amplitude of $1 \mu \mathrm{m}$ whereas the rest of the plate stays at a negligible amplitude.

function or FRF (ratio of the displacement at a given point to the voltage applied to an actuator in the frequency domain) for the actuator. Fig. 4 shows the FRF of the actuator at its center point (in dark blue) at a point $20 \mathrm{~mm}$ away from the actuator on the $x$-axis (in light gold). The FRFs at other points were also measured and show the same behavior. In Fig. 4, the FRF is normalized by the amplitude at $2.9 \mathrm{kHz}$ at the center of the actuator.

At low frequency, these amplitudes exhibit a frequency evolution very similar to those obtained in Section II-B. A non-propagative frequency range appears in the FRF between 0 and $2.9 \mathrm{kHz}$. In this range, the displacement at the center of the actuator is about $30 \mathrm{~dB}$ higher than at any other point on the plate. A representation of the plate at $300 \mathrm{~Hz}$ is represented in Fig. 5. The chosen frequency is below the cut-off frequency, as such, a localized effect corresponding to the non-propagation of modes can be seen. Some points of interest can be highlighted. On Fig. 5, we can discern three areas: the inner surface of the actuator, the outer area near the edge of the actuator, and the rest. Before the cut-off frequency, the spatial deformation inside the actuator depends exclusively on its geometry and nature. The exponentially decreasing shape found in the outer area near the edge of the actuator corresponds to the non-propagative behavior of the plate before its first cut-off frequency.

Following sections aim to verify that the non-propagative 


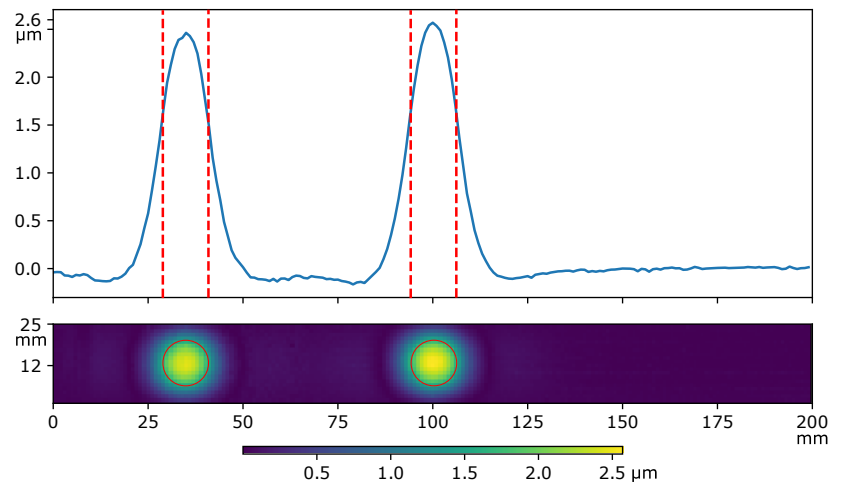

Fig. 6. Measured displacement across the plate. Both vibrations sources are localized and reach a vibration amplitude of $2.6 \mu \mathrm{m}$ while the rest of the plate stays at rest. Outside the actuators, only evanescent waves are present and the amplitude exponentially decreases with the distance from the sources.

behavior of the plate is still relevant when we use multiple actuators and different types of actuators.

\section{B. Simultaneous piezoelectric actuators}

In this section, the possibility to produce multiple located vibrations at the same time thanks to an array of actuators is shown. On the same prototype as before, we added another actuator, identical to the previous one, randomly placed around $7 \mathrm{~cm}$ away from the first actuator. Then we drove both actuators with the same amplitude and frequency. Since the non-propagative frequency band spreads over several $\mathrm{kHz}$ different frequency within this band can be chosen. We chose a sine signal of frequency $300 \mathrm{~Hz}$, which is close to the easiest frequency to feel [22], and a driving voltage amplitude of a $100 \mathrm{~V}$ peak-to-peak for both actuators. The vibration field was measured over the plate by steps of $1 \mathrm{~mm}$. Fig. 6 shows the localized effect. Two distinct excitation sources can be seen. The displacement above each actuator is $2.6 \mu \mathrm{m}$, which is enough for tactile perception at $300 \mathrm{~Hz}$ as specified in [22] and verified in section IV.

\section{Other configurations}

In this section, we prove that the actuator geometry or the nature of the vibration source gives a localized effect as long as the excitation frequency is within the non-propagative range.

A second setup similar to the previous one was implemented: a $200 \times 25 \times 0.5 \mathrm{~mm}^{3}$ aluminum plate clamped on its longer sides to a rigid frame and left free on both ends. We glued on this plate three actuators: two rectangular piezoelectric actuators (PIC255) with different dimensions and a vibration motor (model 306-109 Precision Microdrives TM). A representation of this setup and the three actuators are presented on Fig. 7. The three actuators were placed randomly on the plate.

The first rectangular piezoelectric actuator of dimension $18 \times 6 \times 0.25 \mathrm{~mm}^{3}$ was glued with its longer side along the $y$-axis. It was powered with a sine signal of $100 \mathrm{~V}$ peakto-peak amplitude and $700 \mathrm{~Hz}$ frequency.
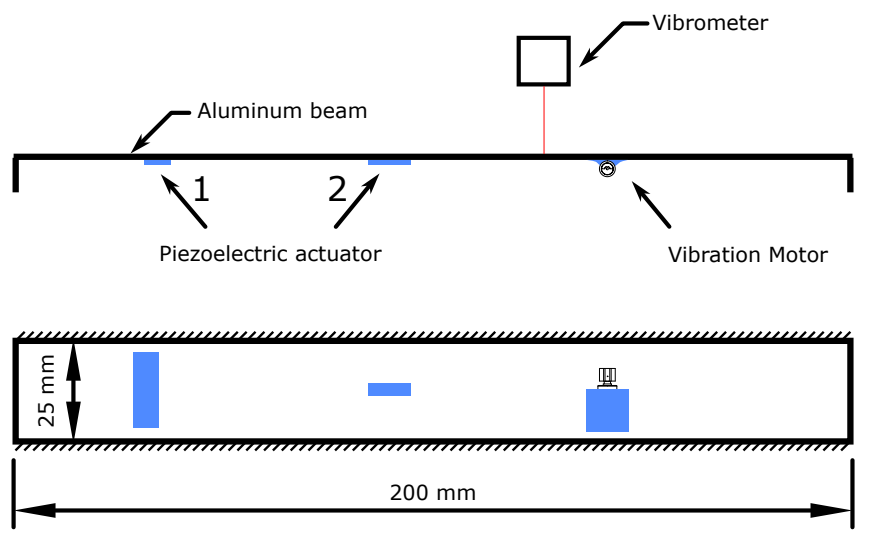

Fig. 7. Experimental setup. Top: Three actuators are glued to the bottom side of an aluminum plate. The laser vibrometer measures the surfaces out of plane displacement. Bottom: plate dimensions and actuator position.

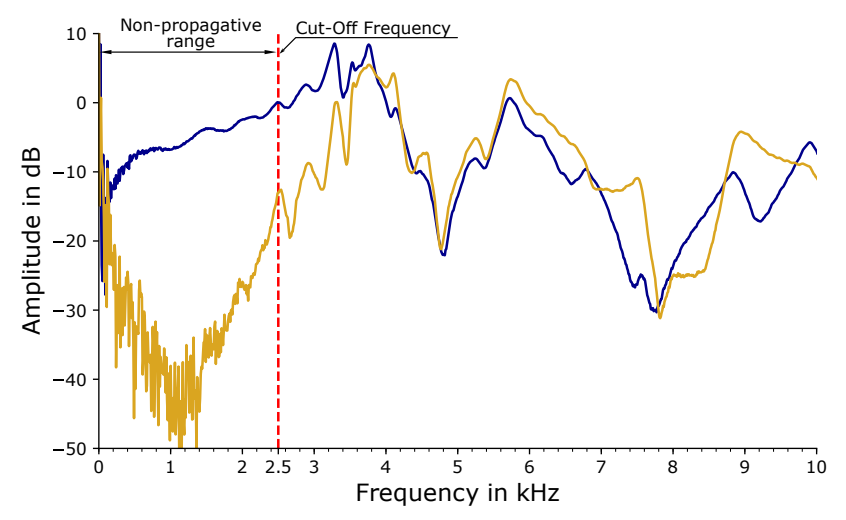

Fig. 8. Measured frequency response function at the center of the actuator (dark blue) and at a point $20 \mathrm{~mm}$ away from the actuator on the $\mathrm{x}$-axis (light gold). The frequency range between $0 \mathrm{~Hz}$ and $2.5 \mathrm{kHz}$ is non-propagative. Within this range, amplitudes at the center of the actuator are about $30 \mathrm{~dB}$ larger than elsewhere on the plate.

The second rectangular piezoelectric actuator, with a dimension of $10 \times 3 \times 0.25 \mathrm{~mm}^{3}$ is four times smaller than the first one. It also differs in orientation as its longer side is along the $\mathrm{x}$-axis. It was also driven with a sine signal of $100 \mathrm{~V}$ pick-to-pick amplitude and $700 \mathrm{~Hz}$ frequency.

The vibration motor was glued to the surface via epoxy resin. The contact geometry is a rectangle of dimension $10 \times 10$ $\mathrm{mm}^{2}$. The vibration motor was driven with a $2 \mathrm{~V}$ continuous signal which gives a dominant frequency around $90 \mathrm{~Hz}$. Before undertaking each experiment, we first realised the FRF of this second plate, as in Section III-A, in order to verify the presence of the non-propagative range. The plate's FRF is represented in Fig. 8. As before, the non-propagative range can be seen extending form from 0 to $2.5 \mathrm{kHz}$ with some slight differences certainly due to the clamping quality and also a possible small difference in plate dimension. For each actuators, we measured the displacement across the plate which are represented on Fig. 9,10 and 11.

For the first actuator (vertical rectangular piezoelectric noted ' 1 ' on Fig. 7.), we can see a maximum amplitude of $2.8 \mu \mathrm{m}$ above the actuator which decreases exponentially 


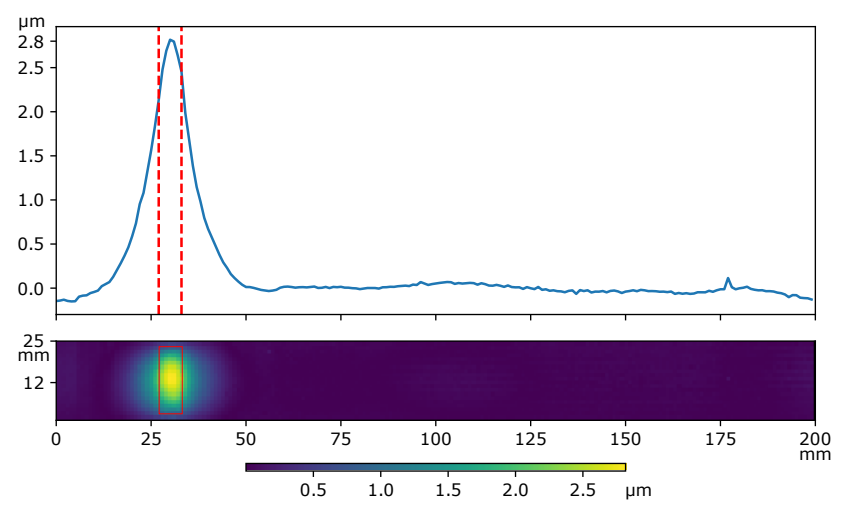

Fig. 9. Measured displacement across the plate excited by a rectangular piezoelectric patch of dimension $18 \times 6 \times 0.25 \mathrm{~mm}^{3}$. A maximum amplitude of $2.8 \mu \mathrm{m}$ is found at the center of the actuator while the rest of the plate stays at rest.

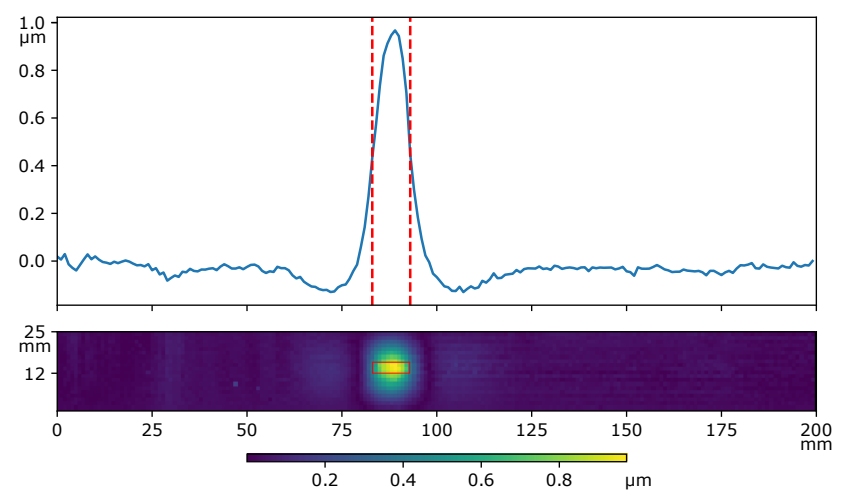

Fig. 10. Measured displacement across the plate excited by a rectangular piezoelectric patch of dimension $10 \times 3 \times 0.25 \mathrm{~mm}^{3}$. A maximum amplitude of $1 \mu \mathrm{m}$ is found at the center of the actuator while the rest of the plate stays at rest.

outside of the actuator covered area. It is interesting to notice the shape of the plate deformation. The rectangular geometry of the actuator gave a seemingly thinner form to the out plane displacement which, in the end, could give a different feedback to the finger [22]. For the second actuator (horizontal rectangular piezoelectric noted '2' on Fig. 7.), we have once again a localized excitation with a maximum amplitude of $1 \mu \mathrm{m}$ at its center. We can see, on the 2D representation of Fig. 10, that even if the ydimension of the actuator is really small that the entire width of the plate is activated which is to be expected because in equation (13) of Section II the y-component is modulated by a sine function. Along the $\mathrm{x}$-axis we find the same effect as before: an amplitude exponentially decreasing with the distance. Finally, the vibration motor also gives us the nonpropagative effect. Fig. 11 shows a maximum displacement of $0.5 \mu \mathrm{m}$. Spikes produced by measurement noise are visible on this figure. The reduced signal to noise ratio in this measurement comes from the lower displacement amplitude and the lower vibration frequency, around $90 \mathrm{~Hz}$. Ambient vibrations indeed have more energy in low frequency and the
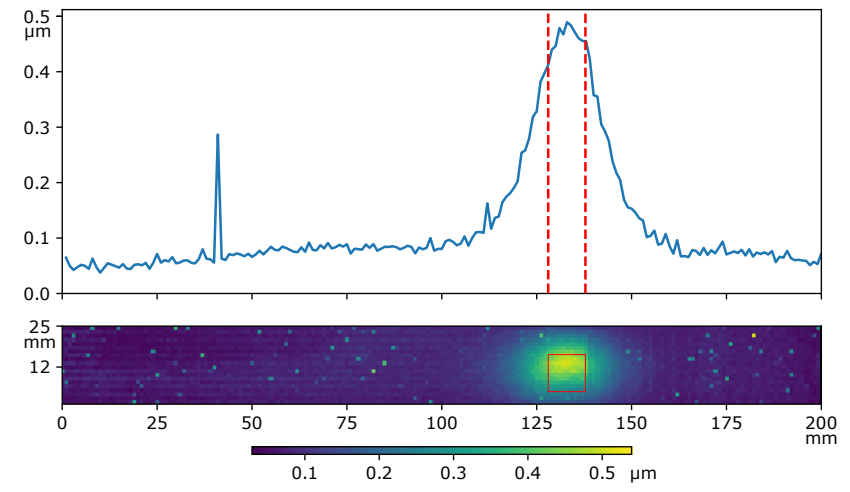

Fig. 11. Maximum displacement measured across the plate excited by a vibration motor with a contact geometry of dimension $10 \times 10 \mathrm{~mm}^{2}$. (The glitch that appears around $40 \mathrm{~mm}$ is due to the defocusing of the laser vibrometer.)

surface velocity measured and integrated by the vibrometer is also lower.

\section{PERCEPTUAL VALIDATION}

A user evaluation was carried out to demonstrate the ability of the device to produce noticeable and localized tactile feedback. This evaluation consisted in a detection task to determine whether the stimulation, with a dynamic signal with transitory and large frequency spectrum, could be felt.

\section{A. Stimuli, Participants, and Procedure}

To demonstrate the wide band capability of our device, we used a one cycle burst sine at $500 \mathrm{~Hz}$. This low number of cycles yields an excitation with $\mathrm{a}-3 \mathrm{~dB}$ bandwidth spreading from $200 \mathrm{~Hz}$ to $700 \mathrm{~Hz}$. The amplitude was changed at every trial by selecting it randomly among five uniformly distributed values ranging from $60 \mathrm{~V}$ to $200 \mathrm{~V}$ peak-topeak. Five volunteers, 1 female and 4 males, aged 20 to 26 participated in the study. Participants had to place both index fingers above each areas covered with piezoelectric actuators of the system depicted in III-B. 75 stimuli were randomly presented at each finger, for a total of 150 stimuli. In addition to these 150, 5 catch trials consisting in exciting both fingers at the same time were added. Each participant had to answer left, right or both. They wore isolating headphones playing pink noise during the whole duration of the task, and could not hear the stimuli.

\section{B. Results and Discussion}

The probability of detection of a stimulus was calculated for each participant and each driving voltage amplitude. This probability is, at a given stimuli amplitude, the ratio of the number of detected stimuli over the number of presented stimuli. Table I show the minimum, maximum and average of detection probability over all participants. As expected, the detection probability increased with driving voltage amplitude. It reached up to 1 for several participants, indicating a high rate of detection of the stimuli. Finally, 24 out of the 25 catch trials sent were noticed showing the multitouch capability of our device. 
TABLE I

PROBABILITY OF DETECTION FOR VARIOUS DRIVING VOLTAGE AMPLITUDES

\begin{tabular}{|r|ccccc|}
\hline $\begin{array}{r}\text { Driving } \\
\text { Voltage }\left(V_{p p}\right)\end{array}$ & 60 & 95 & 130 & 165 & 200 \\
\hline$[\min ; \max ]$ & {$[0.45 ; 0.9]$} & {$[0.9 ; 1]$} & {$[0.9 ; 1]$} & {$[0.96 ; 1]$} & {$[0.93 ; 1]$} \\
\hline mean & 0.86 & 0.87 & 0.96 & 0.9 & 0.96 \\
\hline
\end{tabular}

\section{DISCUSSION}

This confinement technique is based solely on the geometry of the waveguide, which makes it very simple to implement. In the case of a piezoelectric array, it is sufficient to send the desired signals to obtain the desired effect. Different actuators were tested and showed a localized effect. Localization effect is only present along the length of the waveguide and even narrow actuators cannot yield localized vibrations in the width of the waveguide. Wide actuators covering the whole width should thus be preferred. The spatial resolution along the waveguide axis is given by both the actuator width and the attenuation rate.

An array of piezoelectric actuators covering the entire surface would allow localized rendering of clicks by applying custom low frequency signals like in [23]. Such application is possible because of the wide range of frequency we can work in. Dynamic signals such as clicks can imply several frequencies, however, as long as those frequencies don't go beyond the cut-off frequency, the generated wave will be confined.

Also, in [2], it is shown that if two fingers are subjected to excitation with an amplitude difference of $20 \mathrm{~dB}$ then a masking effect takes place and a stimulation will only be perceived at the finger subjected to the highest vibration. In our setup, we indeed verified that when two fingers are side by side, a stimulus is felt only on the finger right on top of the actuated area. With the exponential decay, a distance as low as $1 \mathrm{~cm}$ is enough to reach the attenuation of $20 \mathrm{~dB}$ required for masking effects on two different hands to take place. This masking effect actually improves the localization of the vibrotactile feedback.

\section{CONCLUSION}

The presented non-propagative phenomenon at low frequency allows the creation of local vibration fields above actuators. By driving the actuators at low-frequencies in the non-propagative frequency range, we can produce local vibrotactile feedback to a set of small regions on the actuated surface directly above the actuator array. Hence, we can provide vibrotactile feedback at different frequencies and amplitudes to multiple fingers or within the same finger at the same time.

Previous work on local friction modulation using nonradiating ultrasonic vibrations [13] can also be implemented here. In the case of an array of piezoelectric glued on a clamped strip, we could create an interface working with both vibrotactile feedback at low frequency and friction modulation at high frequency hence giving a full range of tactile experiences.

\section{REFERENCES}

[1] M. Fukumoto and T. Sugimura, "Active click: tactile feedback for touch panels," in CHI'01 Extended Abstracts on Human Factors in Computing Systems. ACM, 2001, pp. 121-122.

[2] J. R. Kim, X. Dai, X. Cao, C. Picciotto, D. Tan, and H. Z. Tan, "A masking study of key-click feedback signals on a virtual keyboard," in Int. Conf. Human Haptic Sensing and Touch Enabled Comput. Appl. Springer, 2012, pp. 247-257.

[3] J. Kildal, "3d-press: haptic illusion of compliance when pressing on a rigid surface," in Int. Conf. Multimodal Interfaces. ACM, 2010, p. 21.

[4] Y. Visell, B. L. Giordano, G. Millet, and J. R. Cooperstock, "Vibration Influences Haptic Perception of Surface Compliance During Walking," PLoS ONE, vol. 6, no. 3, p. e17697, Mar. 2011.

[5] E. Vezzoli, T. Sednaoui, M. Amberg, F. Giraud, and B. LemaireSemail, "Texture Rendering Strategies with a High Fidelity - Capacitive Visual-Haptic Friction Control Device," in Haptics: Perception, Devices, Control, and Applications, ser. Lecture Notes in Computer Science, F. Bello, H. Kajimoto, and Y. Visell, Eds. Springer International Publishing, 2016, pp. 251-260.

[6] M. Bai and Y. Tsai, "Impact localization combined with haptic feedback for touch panel applications based on the time-reversal approach," J. Acoust. Soc. Am., vol. 129, p. 1297, 2011.

[7] C. Hudin, J. Lozada, and V. Hayward, "Localized Tactile Feedback on a Transparent Surface through Time-Reversal Wave Focusing," IEEE Trans. Haptics, vol. 8, no. 2, pp. 188-198, Apr. 2015.

[8] S. Wöckel, U. Steinmann, and H. Arndt, "Haptics by time reversal of elastic waves," in 2016 IEEE Int. Ultrason. Symp., Sept. 2016, pp. $1-3$.

[9] C. Hudin and S. Panëels, "Localisation of vibrotactile stimuli with spatio-temporal inverse filtering," in Int. Conf. Human Haptic Sensing and Touch Enabled Comp. Appl. Springer, 2018, pp. 338-350.

[10] K. Katumu and J. L. Gorlewicz, "Using modal superposition for generating localized tactile effects on variable friction touchscreens," in 2016 IEEE Haptics Symp. (HAPTICS), Apr. 2016, pp. 211-216.

[11] E. Enferad, C. Giraud-Audine, F. Giraud, M. Amberg, and B. LemaireSemail, "Differentiated haptic stimulation by modal synthesis of vibration field," in 2018 IEEE Haptics Symp. (HAPTICS), Mar. 2018 pp. 216-221.

[12] T. Hoshi and H. Shinoda, "Airborne Ultrasound Tactile Display," in Pervasive Haptics: Science, Design, and Application, H. Kajimoto, S. Saga, and M. Konyo, Eds. Tokyo: Springer Japan, 2016, pp. 121138.

[13] C. Hudin, "Local friction modulation using non-radiating ultrasonic vibrations," in 2017 IEEE WHC, June 2017, pp. 19-24.

[14] V. V. Krylov and F. J. B. S. Tilman, "Acoustic 'black holes' for flexural waves as effective vibration dampers," J. Sound and Vibration, vol. 274, no. 3, pp. 605-619, July 2004

[15] M. Wiertlewski, R. Fenton Friesen, and J. E. Colgate, "Partial squeeze film levitation modulates fingertip friction," Proc. Nat. Academy of Sci., vol. 113, no. 33, pp. 9210-9215, Aug. 2016.

[16] R. P. Feynman, R. B. Leighton, M. Sands, M. A. Gottlieb, and R. Leighton, Eds., The Feynman lectures on physics, definitive ed. ed. San Francisco, Calif.: Pearson, Addison-Wesley, 2006.

[17] S. Gibilisco and N. Sclater, Encyclopedia of electronics. Tab Professional and Reference Books, 1990.

[18] "Optical Waveguides," in Fundamentals of Semiconductor Lasers, ser. Springer Series in Optical Sciences, T. Numai, Ed. New York, NY: Springer New York, 2004, pp. 43-56.

[19] L. A. Belov, S. M. Smolskiy, and V. N. Kochemasov, Handbook of RF, Microwave, and Millimeter-wave Components. Artech House, 2012.

[20] I. Hunter, Theory and Design of Microwave Filters. IET, 2001.

[21] K. F. Graff, Wave motion in elastic solids. New York: Dover Publications, 1991, oCLC: 829198880.

[22] R. T. Verrillo, "Effect of Contactor Area on the Vibrotactile Threshold," J. Acoust. Soc. Am., vol. 35, no. 12, pp. 1962-1966, Dec. 1963.

[23] S. Kim and G. Lee, "Haptic feedback design for a virtual button along force-displacement curves," in Proc. 26th апnи. ACM symp. User interface soft. and technol. ACM, 2013, pp. 91-96. 